\title{
Screening strategies for the diagnosis of asymptomatic Leishmania infection in dialysis patients as a model for kidney transplant candidates
}

\author{
Giorgia Comai ${ }^{1} \cdot$ Alessandra Mistral De Pascali $^{2} \cdot$ Marco Busutti $^{1} \cdot$ Silvia Morini $^{3} \cdot$ Margherita Ortalli $^{3} \cdot$ Diletta Conte $^{1}$. \\ Maria Carla $\mathrm{Re}^{2,3} \cdot$ Gaetano La Manna' ${ }^{1}$ Stefania Varani ${ }^{2,3}$
}

Received: 23 November 2019 / Accepted: 14 January 2020 / Published online: 30 January 2020

(c) The Author(s) 2020

\begin{abstract}
Despite being considered a tropical disease, visceral leishmaniasis (VL) caused by L. infantum is also endemic in the Mediterranean Europe and represents an increasing cause of morbidity and mortality in solid organ transplant (SOT) recipients. VL occurring in kidney transplant recipients is a severe event, often worsening the renal damage and leading to poor outcome. It is believed that most of VL cases in transplant recipients are caused by reactivation of a pre-existent, dormant leishmanial infection induced by the immunosuppressive drugs. Nevertheless, the prevalence of asymptomatic Leishmania infection in candidates to kidney transplant residing in or visiting endemic areas is unknown. As L. infantum is highly circulating in northeastern Italy, we aimed to examine the occurrence of this parasitic infection in 119 dialysis patients living in the mentioned area, 71 of whom were potential candidates to kidney transplant. By employing a combination of sensitive serological and molecular methods, we observed a prevalence of $15.9 \%$ asymptomatic Leishmania infection in the study cohort. This finding emphasizes the need of further evaluating potential screening strategies for Leishmania infection in solid organ transplant candidates residing in or visiting endemic areas.
\end{abstract}

Keywords Leishmania infantum $\cdot$ End stage renal disease $\cdot$ Dialysis treatment $\cdot$ Serological methods $\cdot$ Molecular methods

\section{Introduction}

Leishmaniasis is a chronic infectious disease caused by protozoa of the genus Leishmania [1]. The parasites are spread through the bite of infected phlebotomine sand flies and mostly target reticuloendothelial cells. The clinical spectrum

Giorgia Comai and Alessandra Mistral De Pascali contributed equally to this work.

Giorgia Comai

giorgia.comai@aosp.bo.it

1 Department of Experimental, Diagnostic and Specialty Medicine (DIMES)-Nephrology, Dialysis and Renal Transplant Unit, St. Orsola-Malpighi Hospital, University of Bologna, Via G. Massarenti 9 (Pad. 15), 40138 Bologna, Italy

2 Department of Experimental, Diagnostic and Specialty Medicine, University of Bologna, Bologna, Italy

3 Unit of Clinical Microbiology, Regional Reference Centre for Microbiological Emergencies (CRREM), St. Orsola-Malpighi University Hospital, Bologna, Italy of disease depends on the parasite species and on the host immune response, varying from asymptomatic infection ( $80-95 \%$ of infected individuals) to three different clinical manifestations (5-20\% of cases): visceral leishmaniasis (VL), cutaneous leishmaniasis and mucosal or mucocutaneous leishmaniasis. VL is a severe disease caused by Leishmania species belonging to the Leishmania donovani complex; in the Mediterranean Europe, VL is caused by $L$. infantum. Immunosuppression may reactivate latent leishmanial infection [2] and among HIV-infected patients the risk of clinical VL is increased by over 100 times. In recent years, an increase of VL cases has also been reported in solid organ transplant (SOT) recipients [3, 4].

Among immunocompromised patients, cutaneous and mucocutaneous leishmaniasis are rare, whereas VL is the dominant clinical presentation [4]. The prevalence of VL among SOT recipients is $0.05-0.9 \%$ in endemic areas [5] and more than $100 \mathrm{VL}$ cases following transplantation-mainly kidney transplant—have been reported worldwide up to 2017 [6]. The classic triad of fever, splenomegaly, and pancytopenia only occur in one third of 
SOT patients, leading to difficulties in diagnosis, in particular, splenomegaly is reported less frequently than in immunocompetent patients $[4,6]$. In kidney transplant recipients, leishmaniasis often includes acute interstitial nephritis with moderate inflammation and infiltration of lymphocytes, plasma cells and macrophages and can lead to graft dysfunction [7]. Moreover, VL in SOT exhibits high relapse and mortality rates [2,3]. The most common cause of death in immunocompromised patients with VL is the presence of concomitant bacterial, viral or fungal infections $[4,8]$.

Currently, screening of the candidates to SOT for Leishmania infection in endemic areas is not recommended [2, $5,9]$, but a strict monitoring is suggested in case a Leishmania-positive recipient is found for potential reactivation of this parasitic infection [6].

The aim of this study was to investigate the presence of clinically silent Leishmania infection in candidates to SOT. We analyzed the prevalence of asymptomatic Leishmania infection by serological and molecular tests in a cohort of patients with end stage renal disease (ESRD) undergoing dialysis treatment, most of whom were potential candidates to kidney transplant. The study was performed in the Bologna province, northeastern Italy, which is an endemic area for human leishmaniasis [10].

\section{Methods}

\section{Study population, setting, and samples}

We conducted a retrospective study including a target population of ESRD adult patients in dialysis treatment. Inclusion criteria were: (1) absence of medical history of VL or cutaneous leishmaniasis, (2) lack of symptoms for VL or suspected lesions for cutaneous leishmaniasis at the time of blood sampling, (3) absence of other infectious diseases at the time of blood sampling, and (4) residence in the Bologna province. Among the 119 patients included for the study (92\% Caucasians, $61.3 \%$ male, $38.7 \%$ female, mean age 70 years), 71 patients $(60 \%)$ were potential candidates to kidney transplant. Serum and whole blood samples were collected from May to December 2016 at the Nephrology, Dialysis and Renal Transplantation Unit, St. Orsola-Malpighi University Hospital (Bologna, Italy) and stored at $-80{ }^{\circ} \mathrm{C}$ until analysis. The samples were subsequently investigated for the presence of anti-Leishmania antibodies and leishmanial DNA. The study was conducted in accordance with the Declaration of Helsinki, and the protocol was approved by the Ethics Committee of the St. Orsola-Malpighi University Hospital (project identification code: 97/2017/O/Tess).

\section{Detection of anti-Leishmania antibodies}

The presence of specific anti-Leishmania $\operatorname{IgG}$ was assessed by Western Blot (WB). All sera were analyzed by a commercially available Leishmania Western Blot IgG (LDBio Diagnostics ${ }^{\circledR}$, Lyon, France) according to the manufacturer's recommendations. WB technique provides detailed antibody responses to the leishmanial 14-kDa and 16-kDa proteins of L. infantum. The presence of specific antibody to 14 and/or p16 proteins was considered as a positive result [11].

\section{Detection of parasitic DNA}

A PCR assay based on kinetoplast (k)DNA amplification was developed for detection of Leishmania DNA in whole blood samples. Nucleic acids were extracted from 100 $\mu \mathrm{L}$ whole blood with NucliSENSeasyMAG (Biomerieux, Marcy l'Etoile, France). DNA was eluted in $50 \mu \mathrm{L}$ of elution buffer. DNA was amplified employing a PCR targeting leishmanial kinetoplast (k)DNA [12]. Primers (15 pmol of RV1 5'-CTTTTCTGGTCCTCCGGGTAGG-3', 15 pmol of RV2 5'-CCACCCGGCCCTATTTTACACCAA-3') were synthesized by PrimmBiotech (Milan, Italy) and 50 pmol of TaqMan probe (FAM-TTTTCGCAGAACGCCCCTACC CGC-TAMRA) were synthesized by IDTDNA (Leuven, Belgium). $\beta 2$-microglobulin Real-time PCR assay was run simultaneously as a control of amplification of the extracted DNA. The Real-time PCR assays were performed using the Real Time PCR Cycler Rotor Gene 3000 (Qiagen, Hilden Germany). Real Time PCR was considered positive for Leishmania DNA when an amplification curve at threshold cycle (tC) below 40 was present.

\section{Statistical analysis}

Descriptive statistics were used to analyze demographic and clinical characteristics. Chi-squared test was used to analyze potential correlation between detection of Leishmania infection and gender. Significance was established at $\mathrm{p}<0.05$.

\section{Results}

We obtained clinical samples from 119 ESRD adult patients in dialysis treatment at the St.Orsola-Malpighi University Hospital (Bologna, Italy). Seventy-one out of 119 patients $(60 \%)$ were potential candidates to kidney transplant. The age of patients ranged from 20 to 94 years, with a mean age of 70, with a male-to-female ratio of 73:46. Most of patients were Caucasian (92\%), with a percentage of $2 \%$ of Asian, $5 \%$ of African and $1 \%$ of unknown origin. The 
main causes of ESRD in the cohort of patients were diabetic nephropathy (28\%) and hypertensive vascular nephropathy (18\%). Twenty-three percent of patients had a nephropathy of unknown origin. Demographic and clinical data of the study cohort are reported in Table 1.

Nineteen out of 119 patients (15.9\%) were positive at the serological and/or molecular test for Leishmania infection; the mean age of positive patients was 69 years and the male-to-female ratio was 14:5. There was no significant correlation between presence of asymptomatic Leishmania infection and gender $(\chi 2=1.45, p$ value $=0.23)$. None of the Asian patients was positive for the parasitic infection, while $33 \%$ of African patients and $15 \%$ of Caucasian patients were positive for at least one Leishmania test. Results of WB analysis showed the presence of specific antibody to 14 and/or $16 \mathrm{kDa}$ proteins, indicating the presence of anti-Leishmania IgG in 17 ESRD patients (14.3-95\% CI 8-20.4\%) (Table 2).
Table 2 Western Blot and Real Time PCR to detect asymptomatic Leishmania infection in patients with end stage renal disease $(\mathrm{n}=119)$

\begin{tabular}{llll}
\hline WB Pos & WB Neg & PCR Pos & PCR Neg \\
\hline No. $(\%)$ & No. $(\%)$ & No. $(\%)$ & No. $(\%)$ \\
$17(14.3)$ & $102(85.7)$ & $3(2.5)$ & $116(97.5)$ \\
\hline
\end{tabular}

WB; Western Blot. PCR; polymerase chain reaction. Pos; positive. Neg; negative. No; number

In 3 patients' sera, both bands were detected, while antibodies against p14 or p16 were found in 13 and 1 serum sample, respectively. Circulating Leishmania kDNA was detected in peripheral blood from 3 out of 119 ESRD patients (2.5-95\% CI $0.3-5.3 \%$ ); only 1 patient showed simultaneous detection of anti-leishmanial $\mathrm{IgG}$ (WB reactivity to $14 \mathrm{kDa}$ protein) and parasitic DNA in peripheral blood. None of the patients
Table 1 Demographic variables and clinical data of patients with end stage renal disease

\begin{tabular}{|c|c|c|c|}
\hline & Total $\left(119 \mathrm{pts}^{\mathrm{a}}\right)$ & $\begin{array}{l}\mathrm{No}^{\mathrm{b}} \text { of positive pts to } \\
\text { Leishmania infection } \\
\text { (19) }\end{array}$ & $\begin{array}{l}\text { No. of negative pts to } \\
\text { Leishmania infection } \\
\text { (100) }\end{array}$ \\
\hline \multicolumn{4}{|l|}{ Age, years (range) } \\
\hline $20-34$ & $2(2 \%)$ & $1(5 \%)$ & $1(1 \%)$ \\
\hline $35-49$ & $8(7 \%)$ & $0(0 \%)$ & $8(8 \%)$ \\
\hline $50-64$ & $23(19 \%)$ & $5(26 \%)$ & $18(18 \%)$ \\
\hline $65-79$ & $51(43 \%)$ & $7(37 \%)$ & $44(44 \%)$ \\
\hline$>80$ & $33(28 \%)$ & $6(32 \%)$ & $27(27 \%)$ \\
\hline Unknown & $2(2 \%)$ & $0(0 \%)$ & $2(2 \%)$ \\
\hline Mean age, years & 70 & 69 & 79 \\
\hline \multicolumn{4}{|l|}{ Gender } \\
\hline Female & $46(39 \%)$ & $5(26 \%)$ & $41(41 \%)$ \\
\hline Male & $73(61 \%)$ & $14(74 \%)$ & $59(59 \%)$ \\
\hline \multicolumn{4}{|l|}{ Origin } \\
\hline Caucasian & $110(92 \%)$ & $17(89 \%)$ & $93(93 \%)$ \\
\hline Asian & $2(2 \%)$ & $0(0 \%)$ & $2(2 \%)$ \\
\hline African & $6(5 \%)$ & $2(11 \%)$ & $4(4 \%)$ \\
\hline Unknown & $1(1 \%)$ & $0(0 \%)$ & $1(1 \%)$ \\
\hline \multicolumn{4}{|l|}{ Cause of ESRD } \\
\hline Diabetic nephropathy & $33(28 \%)$ & $4(21 \%)$ & $29(29 \%)$ \\
\hline Glomerular disease & $13(11 \%)$ & $2(11 \%)$ & $11(11 \%)$ \\
\hline Hypertensive vascular nephropathy & $21(18 \%)$ & $6(32 \%)$ & $15(15 \%)$ \\
\hline Interstitial disease & $16(13 \%)$ & $1(5 \%)$ & $15(15 \%)$ \\
\hline Polycystic kidney disease & $9(8 \%)$ & $1(5 \%)$ & $8(8 \%)$ \\
\hline Unknown & $27(23 \%)$ & $5(26 \%)$ & $22(22 \%)$ \\
\hline \multicolumn{4}{|l|}{ Dialysis vintage, years (range) } \\
\hline $0-3$ & $47(39 \%)$ & $10(53 \%)$ & $37(37 \%)$ \\
\hline $4-7$ & $35(29 \%)$ & $4(21 \%)$ & $31(31 \%)$ \\
\hline $7-10$ & $17(14 \%)$ & $2(11 \%)$ & $15(15 \%)$ \\
\hline$>10$ & $20(17 \%)$ & $3(16 \%)$ & $17(17 \%)$ \\
\hline
\end{tabular}

Percentages are calculated based on the total number for each column ${ }^{\mathrm{a}}$ Pts; patients, ${ }^{\mathrm{b}} \mathrm{No}$; number 
testing positive to anti-leishmanial antibody and/or parasitic DNA showed signs or symptoms compatible with VL or tegumentary leishmaniasis.

\section{Discussion}

To our knowledge, there are no studies on the prevalence of asymptomatic Leishmania infection in kidney transplant candidates. Here, we detected the presence of clinically silent Leishmania infection in $15.9 \%$ of ESRD patients in dialysis treatment. As $60 \%$ of these patients were potential recipients for kidney transplant, this study group might be considered as a model for candidates to SOT.

Leishmania-specific antibodies were revealed by WB in serum samples obtained from 17 patients, which showed antibody to $14 \mathrm{and} /$ or $16 \mathrm{kDa}$ proteins, while parasitic DNA was detected in blood samples of 3 patients by kDNA amplification. Overall, our results suggest an important cumulative exposure to Leishmania in the examined cohort, which may potentially lead to parasitic reactivation upon transplantation.

A recent study from southern Spain showed a prevalence of $4.8 \%$ of asymptomatic Leishmania infection in renal transplant recipients [13]; differently from this study, we enrolled dialyzed patients who did not receive immunosuppressive drugs. The lower prevalence of Leishmania infection observed in the Spanish study as compared to our study can be related to a variable circulation of the parasite in different endemic areas or to diversity of methods employed for parasite screening. Considering the latter possibility, the indirect immunofluorescence assay (IFAT) employed by Elmahallawy and colleagues could have underestimated the infection rate in asymptomatic carriers. In fact, evidence indicates that IFAT exhibits a lower sensitivity than WB and sera from VL patients recognize the $14-16-\mathrm{kD}$ bands in WB at concentrations $10^{7}$ lower than the threshold values of IFAT [11]. As using a combination of tests has been reported to increase the capacity to detect asymptomatic Leishmania infection [1], we employed the most sensitive serological method, namely WB $[9,11,14]$ together with a molecular test.

Leishmania infection may be transmitted to transplant recipients by different ways, including the donor allograft, the transfusion of blood products, a de novo posttransplant infection or through reactivation of pretransplant infection, the latter generally considered the most common route of infection $[2,15]$. Nevertheless, studies focusing on parasite route of transmission in SOT are lacking. In addition, there is no consensus recommendation to screen the organ transplant recipients for Leishmania infection in endemic areas $[2,5,9]$. Furthermore, different methods are available to detect asymptomatic Leishmania infection, including serology, molecular tests and ex vivo whole blood stimulation with parasitic antigens combined with specific cytokine release assay $[1,16]$, but no gold standard method has been defined so far $[5,17]$. On the other hand, updated guidelines recommend that those known to be Leishmania-seropositive at the time of transplant should be monitored closely for potential reactivation of the parasitic infection [5].

Considering the high prevalence of Leishmania infection among potential kidney transplant recipients detected in the current study, we emphasize the importance of further evaluating sensitive tools for screening this hemoflagellate protozoan in SOT candidates in endemic areas.

Acknowledgements This work was supported by the University of Bologna (RFO funds to SV, MCR and GLM), and by the Italian Ministry of Health (Ricerca Finalizzata RF-2016-02361931 to SV).

Author contributions Gorgia Comai, Stefania Varani, Gaetano La Manna and Maria Carla Re contributed to the study conception and design. Material preparation, data collection and analysis were performed by Alessandra Mistral De Pascali, Diletta Conte, Silvia Morini, Margherita Ortalli and Marco Busutti. The first draft of the manuscript was written by Stefania Varani, Alessandra Mistral De Pascali and Giorgia Comai, and all authors commented on previous versions of the manuscript.

Funding Funding by the University of Bologna (projects RFO "Ricerca Fondamentale Orientata" managed by SV, MCR and GLM), and by the Italian Ministry of Health (project Ricerca Finalizzata RF-201602361931 managed by SV).

\section{Compliance with ethical standards}

Conflict of interest On behalf of all authors, the corresponding author states that there is no conflict of interest.

Ethical approval The study was approved by the local Institutional Ethics Committee.

Informed consent Informed consent was obtained from all individual participants included in the study in accordance with 1964 Helsinki Declaration.

Open Access This article is licensed under a Creative Commons Attribution 4.0 International License, which permits use, sharing, adaptation, distribution and reproduction in any medium or format, as long as you give appropriate credit to the original author(s) and the source, provide a link to the Creative Commons licence, and indicate if changes were made. The images or other third party material in this article are included in the article's Creative Commons licence, unless indicated otherwise in a credit line to the material. If material is not included in the article's Creative Commons licence and your intended use is not permitted by statutory regulation or exceeds the permitted use, you will need to obtain permission directly from the copyright holder. To view a copy of this licence, visit http://creativecommons.org/licenses/by/4.0/. 


\section{References}

1. Gradoni L, Lopez-Velez R, Mokni M (2017) Manual on case management and surveillance of the leishmaniases in the WHO European Region. World Health Organization. https://www.euro. who.int/en/publications/abstracts/manual-on-case-management -and-surveillance-of-the-leishmaniases-in-the-who-europeanregion-2017 Accessed 1 Oct 2019

2. van Griensven J, Carrillo E, López-Vélez R, Lynen L, Moreno J (2014) Leishmaniasis in immunosuppressed individuals. Clin Microbiol Infect 20:286-299

3. Antinori S, Cascio A, Parravicini C, Bianchi R, Corbellino M (2008) Leishmaniasis among organ transplant recipients. Lancet Infect Dis 8:191-199

4. Alves da Silva A, Pacheco-Silva A, de Castro Sesso R, Esmeraldo RM, Costa de Oliveira CM, Fernandes PF et al (2013) The risk factors for and effects of visceral leishmaniasis in graft and renal transplant recipients. Transplantation 95(5):721-727

5. La Hoz RM, Morris MI, Infectious Diseases Community of Practice of the American Society of Transplantation (2019) Tissue and blood protozoa including toxoplasmosis, Chagas disease, leishmaniasis, Babesia, Acanthamoeba, Balamuthia, and Naegleria in solid organ transplant recipients- Guidelines from the American Society of Transplantation Infectious Diseases Community of Practice. Clin Transplant 33(9):e13546

6. Gajurel K, Dhakal R, Deresinski S (2017) Leishmaniasis in solid organ and hematopoietic stem cell transplant recipients. Clin Transplant. https://doi.org/10.1111/ctr.12867

7. Clementi A, Battaglia G, Floris M, Castellino P, Ronco C, Cruz DN (2011) Renal involvement in leishmaniasis: a review of the literature. NDT Plus 4(3):147-152

8. Clemente W, Vidal E, Girão E, Ramos AS, Govedic F, Merino E et al (2015) Risk factors, clinical features and outcomes of visceral leishmaniasis in solid-organ transplant recipients: a retrospective multicenter case-control study. Clin Microbiol Infect 21:89-95

9. Michel G, Pomares C, Ferrua B, Marty P (2011) Importance of worldwide asymptomatic carriers of Leishmania infantum (L. chagasi) in human. Acta Trop 119:69-75
10. Varani S, Cagarelli R, Melchionda F, Attard L, Salvadori C, Finarelli AC et al (2013) Ongoing outbreak of visceral leishmaniasis in Bologna Province, Italy, November 2012 to May 2013. Euro Surveill 18:20530

11. Mary C, Lamouroux D, Dunan S, Quilici M (1992) Western blot analysis of antibodies to Leishmania infantum antigens: potential of the 14-kD and 16-kD antigens for diagnosis and epidemiologic purposes. Am J Trop Med Hyg 47:764-771

12. Mary C, Faraut F, Lascombe L, Dumon H (2004) Quantification of Leishmania infantum DNA by a real-time PCR assay with high sensitivity. J Clin Microbiol 42:5249-5255

13. Elmahallawy EK, Cuadros-Moronta E, Liébana-Martos MC, Rodríguez-Granger JM, Sampedro-Martínez A, Agil A et al (2015) Seroprevalence of Leishmania infection among asymptomatic renal transplant recipients from southern Spain. Transpl Infect Dis 17:795-799

14. Biglino A, Bolla C, Concialdi E, Trisciuoglio A, Romano A, Ferroglio E (2010) Asymptomatic Leishmania infantum infection in an area of northwestern Italy (Piedmont region) where such infections are traditionally nonendemic. J Clin Microbiol 48:131-136

15. Postorino MC, Bellantoni M, Catalano C, Caridi G, De Rosa M, Seck $S$ et al (2011) Visceral leishmaniasis reactivation in transplant patients: a minireview with report of a new case. J Nephrol 24:530-534

16. Carrillo E, Carrasco-Antón N, López-Medrano F, Salto E, Fernández L, San Martín JV et al (2015) Cytokine release assays as tests for exposure to leishmania, and for confirming cure from leishmaniasis, in solid organ transplant recipients. PLoS Negl Trop Dis 9:e0004179

17. Singh OP, Hasker E, Sacks D, Boelaert M, Sundar S (2014) Asymptomatic leishmania infection: a new challenge for leishmania control. Clin Infect Dis 58:1424-1429

Publisher's Note Springer Nature remains neutral with regard to jurisdictional claims in published maps and institutional affiliations. 